\title{
Positive contrast as due to happiness
}

\author{
LAWRENCE WEINSTEIN \\ Brandon University, Brandon, Manitoba R7A 6A9, Canada
}

\begin{abstract}
Upward incentive contrast effects that occurred in people who were prone to be happy in reply to their environment were not observed in other, less "happy-prone" individuals. These data supply needed information on an appetitive emotional account of positive contrast.
\end{abstract}

Positive incentive contrast effects occur when an increment in incentive magnitude produces a significant increase in performance above a control level of behavior that has been maintained on the larger magnitude of reward throughout training. While the phenomenon is now well documented (e.g., Mellgren, 1972; Shanab, Birnbaum, \& Cavallaro, 1974; Weinstein, 1970, 1971, 1980 ), very little is known as to what causes the effect. The present study examined the notion that upward contrast is due to some appetitive emotional or motivational state, such as happiness. If an increment in amount of reward produces a positive emotional state that energizes behavior and so produces upward contrast, then one would expect that individuals who are more sensitive to the facilitating (energizing) effects of happiness would display larger contrast effects than would people who are less likely to respond in a happy fashion to a change in an environmental event. Such data would be consonant with the idea that upward contrast with humans is due to an appetitive or pleasant emotional state. The current experiment examined the effects of an increment in amount of reward in happier and less happy people.

\section{METHOD}

\section{Subjects}

The subjects, 60 males, attended St. Ambrose, St. Leo, and Yankton College. Thirty subjects were assigned randomly to each of two equal groups, a happy group and an unhappy group. The 30 happy people were chosen from 120 subjects, and the remaining 30 individuals were also selected from the same 120 subjects. All 120 people initially chatted with the experimenter, who counted, in a 45-min session, how many times each subject smiled and laughed. A smile could occur with or without a laugh. If a subject in one session smiled 30 times or more and laughed 10 times or more (both criteria had to be met for each happy subject), he was classified a happy subject; if a subject smiled 20 or fewer times and also laughed 3 or fewer times in one session, he was selected as an unhappy person. After chatting with the experimenter, subjects in both groups (50 and 55 people) were administered a type of rating scale in which each person rated himself from 0 to 50 points as to how happy he felt during the discussion with the

Requests for reprints should be sent to Dr. L. Weinstein, Department of Psychology, Brandon University, Brandon, Manitoba R7A 6A9, Canada. experimenter, 50 being most happy and 0 least happy. Of the 50 happy people, 37 rated 35 or above; of the 37 people, 15 were randomly assigned to a happy/low-reward group (HL), and the same number of happy people (who rated 35 or above) were randomly allocated to a happy/high-reward group (HH). Of the 55 unhappy people, 39 rated themselves 20 or below (were unhappy). Fifteen of the 39 unhappy people were allocated in a random fashion, and in the same numbers as above, to the lowreward (UL) and high-reward (UH) groups. Randomization occurred within each group, happy and unhappy.

\section{Apparatus and Procedure}

The apparatus and essential procedure have been described elsewhere (Weinstein, 1972). In a verbal reaction time task, a slide projector was used to present 20 mental multiplication problems (three-digits multiplied by one digit) to each subject; one correct or incorrect answer, or $60 \mathrm{sec}$, whichever came first, was allowed for each problem.

The groups worked the problems in the following ways: (1) Subjects in the HH group received 10 cents after responding to the 2 nd, 3rd, 4th, 6th, 9th, 10th, 11th, 12th, 15th, 17th, and 18th problems; (2) the HL people received 1 cent through Problem 10 and then experienced an increment in incentive size to 10 cents on Problem 11 and on the remaining problems designated above; (3) UH individuals received the same treatment as HH people; and (4) UL subjects experienced the same procedure as the HL group.

The experimenter manually administered the rewards.

\section{RESULTS}

Regarding the rating scale analysis, the means for the two groups, happy and unhappy, differed statistically by an analysis of variance $[F(1,58)=4.91$, $\mathrm{p}<.05]$. The mean for the happy people was 36.6 , and that for the unhappy individuals was 18.5.

Latency means (the time between slide onset and the first reply) were examined in the analysis of the results.

From Figure 1, it seems that from Problems 1-11, the UL subjects reacted slower than the UH subjects and the HL subjects acted slower than the HH subjects. The mean latency from Problems 1-11 differed statistically among the four groups by an analysis of variance $[F(3,56)=4.71, p<.01]$. By Newman-Keuls multiple comparisons (Winer, 1971, p. 191), the differences between $\mathrm{HL}$ and $\mathrm{HH}$ and between UL and $\mathrm{UH}$ were each statistically significant $(p<.05)$, whereas the differences between HL and UL and between $\mathrm{HH}$ and UH were each not statistically valid $(p>.05)$. 


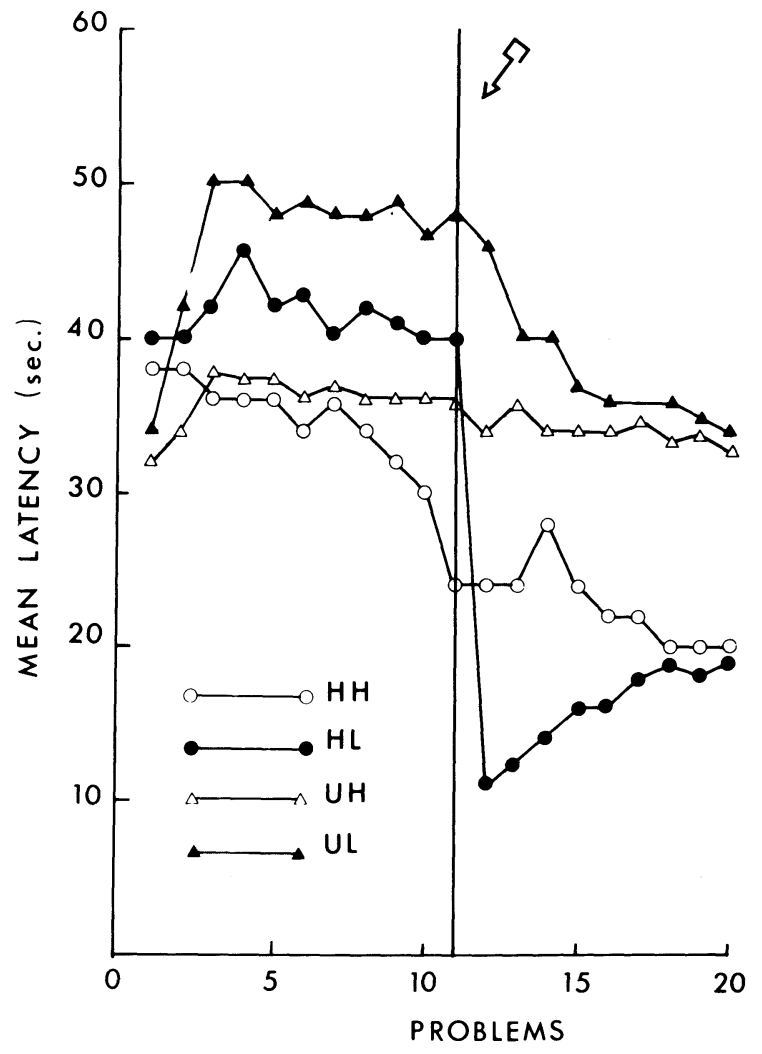

Figure 1. Mean latency of first (correct or incorrect) response. Arrow denotes that after responding to the 11th problem, reward magnitude was increased for the first time.

Figure 1 indicates that on Problem 12 the HL group took less time to reply than the $\mathrm{HH}$ group (positive incentive contrast effects), whereas the UL people did not reply quicker on Problem 12 than the appropriate control group, UH. The mean latency over Problems 12-20 differed significantly among the four groups by an analysis of variance $[\mathrm{F}(3,56)=3.43$, $\mathrm{p}<.05]$. By Newman-Keuls comparisons, the difference between $\mathrm{HL}$ and $\mathrm{HH}$ was significant $(\mathrm{p}<.05)$, whereas the difference between UL and $\mathrm{UH}$ was not statistically significant $(\mathrm{p}>.05)$.

\section{DISCUSSION}

The finding that there were rating scale differences between the frequent smilers and laughers and the less frequent smilers and laughers supports the conviction that the two groups were discrepant in terms of happiness as opposed to some other trait.

For $\mathrm{H}$ and $\mathrm{U}$ conditions, each level of reward was discriminably different (HH vs. HL, and UH vs. UL). Levels of reward here are defined as being different in terms of the statistically different levels of behavior that are produced, rather than in terms of the stimulus scale ( 1 cent vs. 10 cents). Furthermore, the lack of significance between levels of happiness for each level of reward leaves one with no reason to suppose that there are acquisition differences on this organismic variable (positive emotionality). That is to say, it is more than conceivable that subjects were selected for happiness and not for some other correlated variable.

The experiment clearly showed that people who smiled and laughed more displayed positive contrast, whereas other individuals who seemed to be less sensitive to respond in a happy fashion to environmental events did not display the upward incentive contrast effects. Such results are consistent with an interpretation of positive contrast as due to a positive polarized motivational state called, perhaps, happiness. These data support the original Crespi (1944) eagerness notion and Capaldi's (1974) reinforcement-level hypothesis. Both notions assert that when expected reward level is less than obtained reward level, positive contrast occurs.

The present results, like the results of almost any investigation dealing with an organismic (subject) variable, are open to the quite reasonable criticism that one must be wary of notions based upon a single dimension of behavior for which organisms are selected. Such an approach is not to be discouraged when correlates of the individual differences are thoroughly examined and accounted for. Future studies looking at this or a similar treatment effect consisting of positive motivation might expand on the present investigation by equating subjects as to possible covariables that may be present along with the subjective factor under study (i.e., reactivity to environmental change).

The present findings and the results of a number of other investigators (e.g., Mellgren, 1972; Shanab et al., 1974; Weinstein, 1980) rather clearly point out that positive incentive contrast effects can be obtained in a variety of organisms, such as rats and humans, with quite different responses, such as gross motor performance (running) and a cognitive task. Finally, these results afford needed empirical findings and attention directed at the positive incentive shift effect, which has been neither extensively examined as to its controlling variables nor elaborately studied to acquire empirical information pertaining to the few theoretical accounts of the positive phenomenon.

\section{REFERENCES}

Capaldi, E. J. Partial reward either following or preceding consistent reward: A case of reinforcement level. Journal of Experimental Psychology, 1974, 102, 954-962.

Crespi, L. P. Amount of reinforcement and level of performance. Psychological Review, 1944, 51, 341-357.

Mellaren, R. L. Positive and negative contrast effects using delayed reinforcement. Learning and Motivation, 1972, 3, 185-193.

Shanab, M. E., Birnbaum, D. W., \& Cavallaro, G. Positive contrast obtained in reacquisition following interpolation of non-reinforced or partially reinforced trials. Learning and Motivation, 1974, 5, 258-271.

Weinste in, L. Magnitude of incentive contrast as a function of amount of verbal reward change. Psychonomic Science, $1970,20,65-66$.

Weinstein, L. Effects of an increment in monetary incentive magnitude on instrumental responding and repeated increases in reward magnitude in humans. Psychonomic Science, 1971, 25, 235-237.

Weinstein, L. Negative contrast with humans as a function of emotionality. Journal of Psychology, 1972, 80, 161-165.

Weinstein, L. The effects of certain vegetables vs. particular meat breakfasts on the magnitude of human positive contrast and self-ratings of positive emotionality. Bulletin of the Psychonomic Society, 1980, 15, 200-202.

WINER, B. F. Statistical principles in experimental design. Montreal: McGraw-Hill, 1971.

(Received for publication November 23, 1981.) 